\title{
Long term follow up of patients with endomyocardial fibrosis: effects of surgery
}

\author{
U Schneider, R Jenni, J Turina, M Turina, O M Hess
}

\begin{abstract}
Aims-To determine the long term outcome of patients with endomyocardial fibrosis and to compare echocardiographic and haemodynamic data before and after ventricular endocardial resection.

Patients-Seventeen patients (11 women and six men; mean age 35.5 years) diagnosed with endomyocardial fibrosis at the University Hospital in Zurich, Switzerland from 1971 to 1995 . Twelve patients $(70 \%)$ had partial obliteration of both ventricles and in five patients $(30 \%)$ the fibrotic lesions were limited to the left ventricle.

Methods-Fourteen of the 17 patients had surgical resection: fibrosis was resected from both ventricles in five patients and from the left ventricle only in nine patients. Ten patients had mitral valve replacement and two had tricuspid valve replacement. Left ventricle endocardial resection was done without reconstruction or replacement of the atrioventricular valve in three patients. Preoperative and postoperatve echocardiographic data were available for 11 patients and haemodynamic data for six patients. Patients were followed up for 0.4-19 years (mean 8.6).
\end{abstract}

Results-Preoperatively four patients were NYHA functional class IV and 10 were class III; postoperatively one patient was class III, seven class II, and six class I. Preoperatively, echocardiography showed obliteration of the left ventricular apex and inflow tract in all patients, which decreased or disappeared after surgery. Left ventricular end diastolic pressure decreased from $25 \mathrm{~mm} \mathrm{Hg}$ before surgery to $14 \mathrm{~mm} \mathrm{Hg}$ after successful resection of the fibrosis. Left ventricular end diastolic volume (normal $93(17) \mathrm{ml} / \mathrm{m}^{2}$ ) increased from $65 \mathrm{ml} / \mathrm{m}^{2}$ to $97 \mathrm{ml} / \mathrm{m}^{2}(\mathrm{p}<0.05)$ after surgery. Ejection fraction was normal preoperatively $(57 \%)$ and decreased slightly (52\%) after surgery. One patient died five months after surgery from heart failure. Four surgically treated patients died during the follow up period: one each from systolic dysfunction, recurrence of endomyocardial fibrosis, pneumonia, and food poisoning. Overall survival was $65 \%$ at five years and $59 \%$ at 10 years; the survival rates of the operated patients was $72 \%$ and $68 \%$, respectively. Only one of the medically treated patients survived longer than three years from diagnosis.

Conclusions - Endomyocardial fibrosis is a rare disease in European countries and is found mainly in women. The clinical picture is characterised by severe congestive heart failure but heart size is only moderately increased. Systolic performance is normal or only slightly depressed despite severe restriction to filling, atrioventricular valve regurgitation or both. Partial obliteration of the right and/or left ventricle may be detected by echocardiography. Endocardial resection with atrioventricular valve replacement is the treatment of choice with appreciable postoperative improvement and 10 year survival of approximately $\mathbf{7 0 \%}$.

(Heart 1998;79:362-367)

Keywords: endomyocardial fibrosis; atrioventricular valve regurgitation; endocardial resection; hypereosinophilic syndrome

Endomyocardial fibrosis was first described in 1948 by Davies in Uganda. ${ }^{1}$ It is an endemic disease, mainly seen in tropical countries, but found rarely in Europe. ${ }^{2-4}$ The basic feature of the disease is the formation of fibrous tissue on the endocardium of the inflow tract of the right or left ventricle or both, and to a lesser extent in the myocardium. Endomyocardial fibrosis may become so prominent that it leads to partial obliteration of one or both ventricles with decreased ventricular distensibility and impaired diastolic filling. ${ }^{5}$ The fibrotic process is usually located at the apex of the affected ventricle and extends to the inflow tract. Involvement of the chordae tendinae is frequent and causes mitral and/or tricuspid regurgitation. Medical treatment is often unsatisfactory and surgery is the treatment of choice. ${ }^{6-10}$ Eosinophils are thought to play a major role in the development of this disease (fibroblast stimulating hormone).$^{13}{ }^{14}$ Paul Ehrlich, in 1879, was the first to recognise blood eosinophils. ${ }^{15}$ This became well known after 1936, when Löffler wrote his article on endocarditis parietalis fibroplastica. ${ }^{16}$ In the early $1970 \mathrm{~s}$ it was suggested that the tropical as well as the sporadic form of endomyocardial fibrosis might be caused by hypereosinophilia. ${ }^{13}$ In patients with hypereosinophilic syndrome the eosinophil count is commonly $>1.5 \times 10^{9} / 1$ often with raised platelet counts ${ }^{14}$ and overproduction of eosinophils in bone marrow biopsies. Raised concentrations of blood coagulation factors and serum immunglobulin can also be found. In most European cases, there is no serological evidence for parasites, allergies or inflammatory diseases, although in some patients eosinophilic leukaemia or chronic inflammatory processes have been described. 
Table 1 Surgical procedures in 14 patients with endomyocardial fibrosis

\begin{tabular}{ll}
\hline Surgery & Number of patients \\
\hline Resection & $9(6 \mathrm{female} / 3$ male $)$ \\
Left ventricle only & $5(4 \mathrm{female} / 1 \mathrm{male})$ \\
Left and right ventricle & $10(4$ biological $/ 6$ mechanical $)$ \\
Valve replacement & 2 (biological $)$ \\
Mitral & 2 \\
Tricuspid & 5 \\
Valve reconstruction & \\
Mitral (Duran ring) & \\
Tricuspid (de Vega procedure) & \\
\hline
\end{tabular}

The present study describes the clinical long term outcome of 17 patients with endomyocardial fibrosis and determines the preoperative and postoperative haemodynamic changes in 14 of the patients.

\section{Methods}

PATIENTS

From 1971 to 1995 endomyocardial fibrosis was diagnosed in 17 patients (11 female, six male) at the authors' institution. The right ventricle was affected in 10 patients (six female, four male) and the left ventricle in all 17 patients. There were 10 Swiss patients and seven from other countries (two from Yugoslavia, two from Tibet, and one each from Italy, Turky, and Poland). The mean age at the time of diagnosis was 35.5 years (range 18-51). Preoperatively four patients were New York Heart Association (NYHA) functional class IV for congestive heart failure and 10 were in class III. Increased eosinophils were found in eight cases (47\%); however, severe changes (40-50\% eosinophils) were observed in only three $(18 \%)$. No specific abnormalities were found in the electrocardiograms: 15 were in sinus rhythm, three showed left bundle branch block, two atrial fibrillation, and two had complete AV block after right ventricular endocardial resection necessitating pacemaker implantation.

\section{ECHOCARDIOGRAPHY}

The most important diagnostic tool for endomyocardial fibrosis is echocardiography. Between 1975 and 1983 M mode echocardiography only was performed (four patients) but later cross sectional echocardiography was also done. Patients were studied in the supine position usually in the right lateral decubitus. Measurements were carried out according to the American Society of Echocardiography. Septal and posterior wall thickness were determined at end diastole, and left ventricular chamber dimensions at end systole and end diastole. Regional wall motion was assessed visually. After 1983, 10 patients were studied by Doppler echocardiography for determination of mitral regurgitation using a real time phased array sector scanner with integrated colour Doppler facilities and a $2.5 \mathrm{MHz}$ transducer for continuous wave Doppler recordings.

\section{CARDIAC CATHETERISATION}

Fourteen patients had cardiac catheterisation; right and left heart catheterisation with determination of cardiac output by the Fick method, biplane left ventricular angiocardiography, and coronary arteriography was done. Left ven- tricular volumes were calculated according to the area-length method. ${ }^{17}$ In six patients preoperative and postoperative measurements were obtained. ${ }^{18}$

SURGICAL TREATMENT

Fourteen of the 17 patients had surgical resection of the left $(n=14)$ and/or right $(n=5)$ ventricle with simultaneous mitral $(n=10)$ and tricuspid valve $(n=2)$ replacement (table 1). In three patients (two female, one male) left ventricular endocardial resection was performed without reconstruction or replacement of the atrioventricular valve. One patient with mitral valve thrombosis was reoperated after 16 years. Ten patients had mitral valve replacement: four with a biological and six with a mechanical prosthesis. Reconstruction of the tricuspid valve was performed in five patients using the de Vega procedure. Two patients had a biological prosthesis implanted in the tricuspid valve. There were no perioperative deaths (up to 30 days after surgery).

\section{Surgical technique}

All operations were performed with bicaval cannulation under cold cardioplegic arrest. Until 1988 crystalloid cardioplegia was used; in 1989 myocardial protection was changed to blood cardioplegia. The right and left atrium were opened in standard fashion and mitral then tricuspid valves as well as the corresponding ventricular cavities were inspected. The typical cause for regurgitation is restricted leaflet motion caused by thickening of the endocardium, which results in the fixation of the papillary muscles and the chordae tendinae to the walls of the ventricular cavity. When no major valvar abnormality was present, the resection of the thickened endocardium was started, working through the mitral then tricuspid valves. A plane of cleavage was easily developed and the resection procedure was performed with alternating sharp and blunt dissection, trying to remove all of the yellowwhite and thickened endocardium. The tips of the papillary muscles were left in place but papillary muscles as well as chordae tendinae were carefully freed from the often dense adhesions that fix the leaflets in a semiopen position. When necessary, additional annuloplasty was performed on the tricuspid and mitral valve to ensure valvar competence. In the case of major valve abnormalities with an extention of the fibrotic process to the chordae tendinae and leaflets, valve replacement was performed.

As a rule both ventricles can be totally freed from the endocardial thickening that usually does not progress into the outflow tract. Typically the fibrotic process is concentrated in the inlet portion and the main chamber, obliterating the ventricular apex, which gives this disease the characteristic appearance in the angiographic and echocardiographic findings. The ventricles were carefully rinsed to remove all the residual material and standard atrial closure was performed. Intravenous heparin was started within three hours after the end of 
Table 2 Echocardiographic data

\begin{tabular}{llllll}
\hline & $\begin{array}{l}\text { Aortic } \\
\text { diameter }(\mathrm{cm})\end{array}$ & $\begin{array}{l}\text { End systolic LA } \\
\text { diameter (cm) }\end{array}$ & $\begin{array}{l}\text { End systolic LV } \\
\text { diameter (cm) }\end{array}$ & $\begin{array}{l}\text { End diastolic LV } \\
\text { diameter (cm) }\end{array}$ & $\begin{array}{l}\text { Systolic } \\
\text { shortening (\%) }\end{array}$ \\
\hline Preop & 2.9 & 5.2 & 3.9 & 5.9 & 35.3 \\
Postop & 3.1 & 4.8 & 4.2 & 5.9 & 34.3 \\
Normal & $2.0-3.7$ & $1.5-4.0$ & $4.4-6.5$ & $4.4-6.5$ & $29-46$ \\
\hline
\end{tabular}

Values are means of 11 patients.

LA, left atrial; LV, left ventricular.

the surgical procedure; oral anticoagulation with coumarin was initiated on the first postoperative day. More recently, $100 \mathrm{mg}$ aspirin have been added to the oral anticoagulation regimen.

STATISTICS

Preoperative and postoperative data were compared by the paired Student's $t$ test. Normal values for $M$ mode echocardiography were used from a published control group without heart disease. ${ }^{18}$ In all tables and figures mean values are given. A $p$ value $<0.05$ was considered significant.
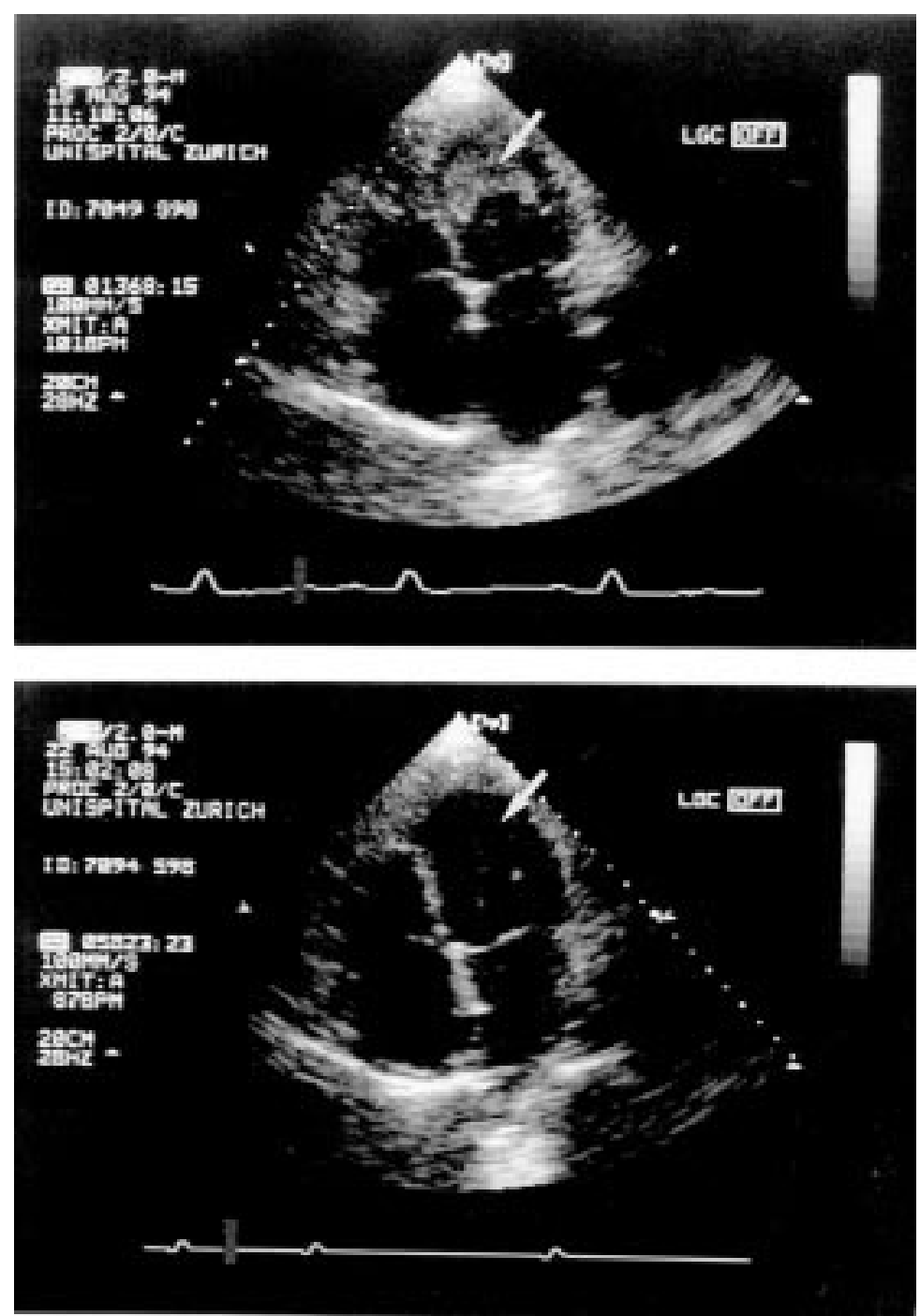

Figure 1 Preoperative (top) and postoperative (bottom) cross sectional echocardiogram (apical four chamber view) of a 52 year old Swiss patient with left ventricular endomyocardial fibrosis and hypereosinophilic syndrome (myeloproliferative disease). The obliteration of the apex (arrow) can be clearly seen. The left and right atria are enlarged owing to the restriction to filling. Postoperatively the obliteration is no longer present (arrow).

\section{Results}

CLINICAL DATA

Medical therapy

Preoperatively, all patients received high doses of diuretics, nine received digitalis, three ACE inhibitors, and seven patients were given anticoagulants. Postoperatively, four patiens were given ACE inhibitors, seven received diuretics, and 11 were given anticoagulants because of valve replacement.

\section{Clinical symptoms}

Postoperatively, one patient was NYHA class III, seven class II, and six class I. There were no operative or perioperative deaths. The only class III patient (male) died within five months of the operation because of biventricular heart failure. The immediate postoperative result of this patient was excellent but there was progressive ventricular dilatation. In two patients with biventricular resection (both female) a pacemaker had to be implanted because of complete AV block. Mean postoperative follow up was 8.6 years; one year mortality in operated patients was $7 \%$, five year mortality $28 \%$, and 10 year mortality $32 \%$.

\section{ECHOCARDIOGRAPHY}

Table 2 shows the mean preoperative and postoperative echocardiographic data for $11 \mathrm{pa}-$ tients.

\section{Preoperative data}

Obliteration of the left ventricular apex and the inflow tract (fig 1) were observed in all patients. Figure 2 shows a representative echocardiographic follow up before and after resection. The left atrium was enlarged in nine of the 11 patients $(82 \%)$ and the right atrium in 10 $(91 \%)$. Dilatation of the left ventricle $(>6.5 \mathrm{~cm})$ was observed in three cases $(27 \%)$. The right ventricle was visually enlarged in seven patients $(64 \%)$. Septal wall thickness was normal $(\leqslant 1.2 \mathrm{~cm})$ in all cases but showed paradoxical wall motion in two patients. Systolic shortening was reduced in three patients (normal 29-46\%).

\section{Postoperative data}

Left $(82 \%)$ and right $(91 \%)$ atrial diameter remained increased postoperatively. After surgical resection, obliteration of the left ventricular apex decreased or disappeared completely in all patients (fig 1). The fibrotic process recurred in one patient 17 months after surgery. Dilatation of the left ventricle was found in four patients (36\%) and of the right ventricle in six patients (55\%). Septal wall thickness remained unchanged postoperatively but there was paradoxical wall motion after surgery in 10 patients $(91 \%)$. Systolic shortening was reduced in two patients but mean systolic shortening was normal preoperatively and postoperatively.

\section{HAEMODYNAMICS}

Table 3 shows the preoperative and postoperative haemodynamic data for six patients. 

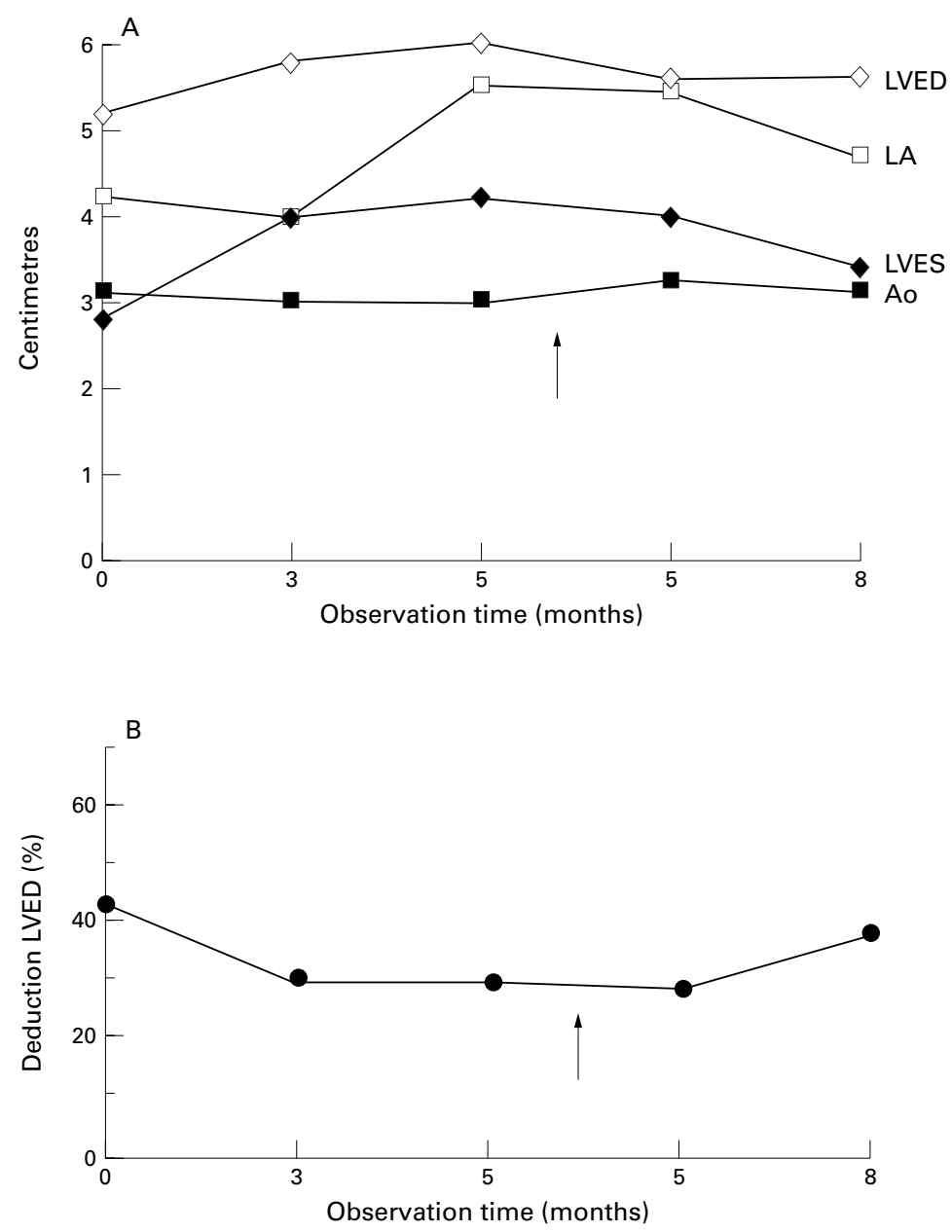

Figure 2 Echocardiographic follow up data in the same patient as fig 1. Arrow indicates time of operation. Preoperatively, there is progressive enlargement of the left atrium $(A)$ and a decrease in systolic shortening (B). Postoperatively, left atrial size decreases and systolic shortening improves. LVED, left ventricular end diastolic diameter; $L A$, left atrial end systolic diameter; LVES, left ventricular end systolic diameter; Ao, aortic diameter.

Preoperative data

Left and right end diastolic pressures were raised preoperatively: left, $25 \mathrm{~mm} \mathrm{Hg}$ (normal $\leqslant 14$ ); right, $10 \mathrm{~mm} \mathrm{Hg}$ (normal $\leqslant 7$ ). Left ventricular ejection fraction was within the lower range of normal: mean 57\% (normal $\geqslant 57 \%$ ), but left ventricular end diastolic volume was reduced: $65 \mathrm{ml} / \mathrm{m}^{2}$ (normal 93 (17)). ${ }^{17}{ }^{19}$

Postoperative data

Left ventricular end diastolic pressure decreased significantly (fig 3) after surgery $(\mathrm{p}<0.05)$ to $14 \mathrm{~mm} \mathrm{Hg}$ but remained raised in half of the patients. Left ventricular systolic

Table 3 Haemodynamic data

\begin{tabular}{llllll}
\hline & $\begin{array}{l}\text { LVSP } \\
(\mathrm{mm} \mathrm{Hg})\end{array}$ & $\begin{array}{l}\text { LVEDP } \\
(\mathrm{mm} \mathrm{Hg})\end{array}$ & $\begin{array}{l}\text { RVEDP } \\
(\mathrm{mm} \mathrm{Hg})\end{array}$ & $\begin{array}{l}E F \\
(\%)\end{array}$ & $\begin{array}{l}E D V I \\
\left(\mathrm{ml} / \mathrm{m}^{2}\right)\end{array}$ \\
\hline Preop & 117 & 25 & 10 & 57 & 65 \\
Postop & 121 & $14^{\star}$ & 9 & 52 & $97^{\star}$ \\
\hline
\end{tabular}

Values are means of six patients.

${ }^{\star} \mathrm{p}<0.05$ compared with preoperative value.

LVSP, left ventricular (LV) peak systolic pressure; LVEDP, LV end diastolic pressure; RVEDP, right ventricular end diastolic pressure; EF, LV ejection fraction; EDVI, LV end diastolic volume.



Figure 3 Left ventricular end diastolic pressure (LVEDP) before and after endocardial resection. There is a significant fall in left ventricular end diastolic pressure after surgery nearly in all patients.

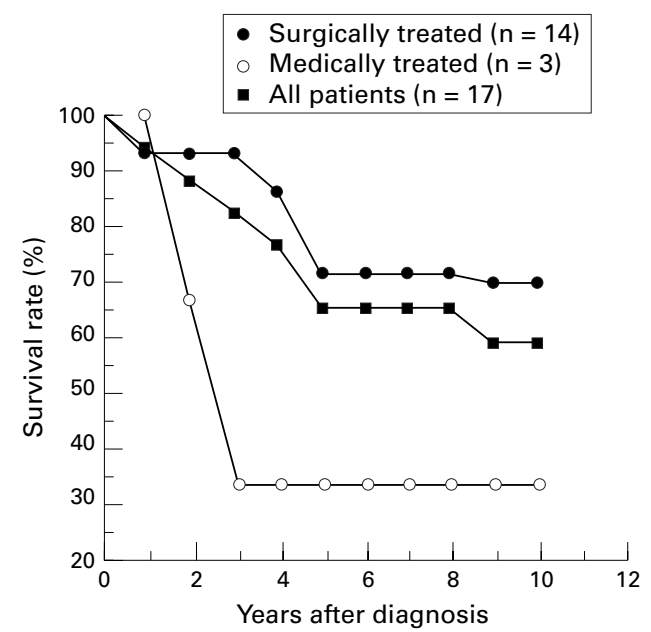

Figure 4 Cumulative survival rates in medically and surgically treated patients with endomyocardial fibrosis. After five and 10 years approximately two thirds of all operated patients were alive, but only one of three medically treated patients.

pressure increased slightly after surgery. There was also a small but insignificant decrease in right ventricular end diastolic pressure. Left ventricular ejection fraction decreased to $52 \%$ after surgery (NS), and left ventricular end diastolic volume increased to $97 \mathrm{ml} / \mathrm{m}^{2}$ $(\mathrm{p}<0.05)$.

\section{FOLLOW UP}

Mean postoperative follow up $(n=14)$ was 8.6 years (range 0.4-19). Four patients (three female, one male) died during follow up $(28 \%)$. Mean cumulative survival rate (fig 4$)$ in all medically and surgically treated patients was $64 \%$ after five years and $59 \%$ after 10 years. However, survival rate in operated patients was $72 \%$ after five years and $68 \%$ after 10 years. Causes of death in operated patients were systolic dysfunction in one, recurrence of endomyocardial fibrosis in one, pneumonia in one, and food poisoning of unknown origin in the patient from Tibet.

\section{Discussion}

Endomyocardial fibrosis is a rare disease in industrialised contries affecting young adults, mainly women $(65 \%)$. Mean age at the time of 


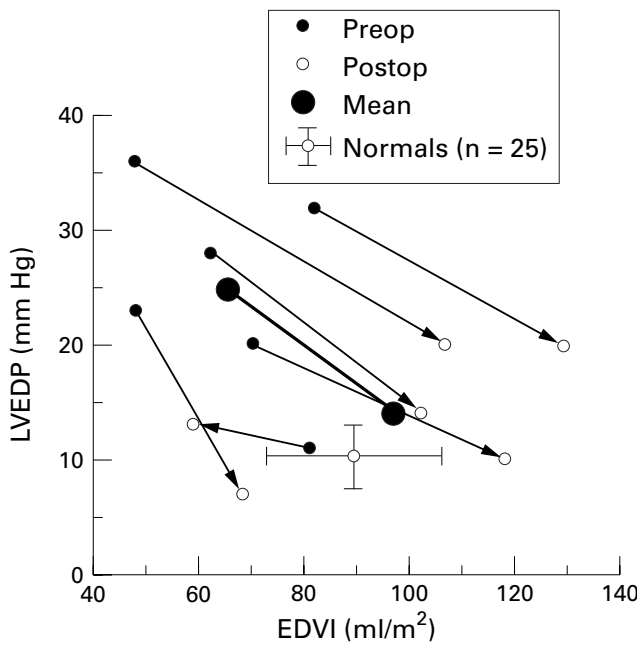

Figure 5 Left ventricular end diastolic pressure (LVEDP) volume (EDVI) relation in six patients before and after endocardial resection. There is a clear tendency for normalisation of LV diastolic dysfunction (high LVEDP and small $L V$ end diastolic volume) after surgical removal of the fibrotic plaque. Normal values are taken from reference 17.

diagnosis was 35.5 years in our population. The fibrotic process typically affects the apex and the inflow tract of one or both ventricles. Severe restrictions to filling are characteristic findings (diastolic dysfunction), whereas systolic pump function is usually maintained. Medical treatment (diuretics, digitalis) is often unsatisfactory and surgery (endocardial resection with $\mathrm{AV}$ valve replacement) is the treatment of choice.

During the past 24 years we observed 17 patients with the sporadic form of endomyocardial fibrosis at our institution. Three patients (18\%) had hypereosinophilic syndrome, ${ }^{23}$ the others showed a chronic progressive course. The severity of the inflow obstruction in 14 of the 17 patients necessitated surgery. Surgical treatment is often the only choice in the presence of severe endomyocardial fibrosis. ${ }^{6-12}$ The 10 year survival rate of the operated patients was $68 \%$. There was a dramatic improvement in quality of life after surgery with a decrease in NYHA functional class for most patients. The fibrosis recurred in one patient 17 months after surgery (acute form, Löffler's endocarditis).

PATHOPHYSIOLOGY

The aetiology of endomyocardial fibrosis is unknown but hypereosinophilia seems to play a major role. ${ }^{14162021}$ A large increase in eosinophils is a typical finding in Löffler's endocarditis, ${ }^{13}$ whereas there is usually no hypereosinophilia in the chronic form (endomyocardial fibrosis). A fibroblast stimulating hormone of eosinophils has been postulated that may induce fibrosis of the ventricles. The cause of hypereosinophilia may vary but is typically Loa-Loa infection in tropical countries, as observed in one of our patients who spent some time in Africa. There may be an interval of several years between the filariasis and the development of the inflow obstruction. ${ }^{22}$
CLINICAL COURSE

Severe filling restrictions may lead to pulmonary congestion and low output failure although systolic pump function may be maintained even in the late course of the disease. However, two of our patients developed severe left ventricular systolic dysfunction after left ventricular endocardial resection and died before transplantation with dilated cardiomyopathy. In one patient the development of systolic dysfunction occurred within five months of surgery, whereas in the other patient it did not develop until 11 years postoperatively. Several operated patients had stable follow up over many years. One year mortality (all cardiac deaths occurring between three months and one year postoperatively) was $7 \%$ and 10 year mortality was $32 \%$. Left ventricular endocardial resection was associated with a dramatic improvement in left ventricular diastolic function (fig 5). Not only did left ventricular end diastolic pressure drop significantly (table 3) but left ventricular end diastolic volume increased-that is, surgery reversed the left ventricular diastolic dysfunction, but medical treatment could not stop the progressive course of this disease. Even in patients with poor prognosis due to the underlying disease (myeloproliferative syndrome) left ventricular resection may lead to a significant improvement in left ventricular function.

\section{CLINICAL IMPLICATIONS}

Long term prognosis is usually poor in patients with endomyocardial fibrosis and is dependent on the aetiology of the underlying disease and the severity of the fibrotic process. The treatment of choice is endocardial resection of one or both ventricles even in patients with rapid progression of the underlying disease. Reversal of diastolic dysfunction with an increase in cardiac output can be observed in most patients, which is associated with an improvement in symptoms. Ten year survival rates of approximately $70 \%$ are realistic in operated patients with endomyocardial fibrosis.

1 Davies JNP. Endomyocardial necrosis. A heart disease of obscure aetiology in Africa. Bristol: MD Thesis, 1948

2 Shaper AG, Hutt MSR, Coles RM. Necropsy study of endomyocardial fibrosis and rheumatic heart disease in Uganda 1960-1965. Br Heart f 1968;30:391-401.

3 Somers K, Hutt MSR, Patel AK, et al. Endomyocardial biopsy in diagnosis of cardiomyopathies. Br Heart $\mathcal{f}$ 1971;33:822-32.

4 Chew CYC, Ziady GM, Raphael MJ, et al. Primary restrictive cardiomyopathy. Nontropical endomyocardial fibrosis and hypereosinophilic heart disease. Br Heart $\mathcal{f}$ 1977;39: and hyper 413 .

5 Hess OM. Endomyokardfibrose. In: Krayenbuehl HP, Kuebler W, eds. Herzkrankheiten. Stuttgart: Thieme Verlag,

6 Dubost Ch, Maurice P, Gerbaux A, et al. The surgical treatment of constrictive fibrous endocarditis. Ann Surg 1976;184:303-7.

7 Cherian G, Vijayaraghavan G, Krishnaswami S, et al. Endomyocardial fibrosis: report on the hemodynamic data in 29 patients and review of the results of surgery. Am Heart f 1983;4:659-65.

8 Hess OM, Turina M, Senning A, et al. Pre- and postoperative findings in patients with endomyocardial fibrosis. $\mathrm{Br}$ Heart f 1978;40:406-15.

9 Metras D, Coulibaly AQ, Quattara K, et al. Endomyocardial fibrosis; early and late results of surgery in 20 patients. $\mathcal{f}$ Thorac Cardiovas Surg 1982;83:52-64.

10 Moraes CR, Buffolo El, Lima R, et al. Surgical treatment of endomyocardial fibrosis. F Thorac Cardiovasc Surg 1983;85: 738-45.

11 Blakrishnan KG, Venkitachalam CG, Pillai VRK, et al. Postoperative evaluation of endomyocardial fibrosis. Cardiology
1986;73:73-84. 
12 Davies J, Sapsford R, Brooksby I, et al. Successful surgical treatment of two patients with eosinophilic endomyocardial treatment of two patients with eosinophi
disease. Br Heart $\mathcal{7} 1981 ; 46: 438-45$.

13 Brockington JF, Olsen EGJ. Löffler's endocarditis and Davies' endomyocardial fibrosis. Am Heart f 1973;85:30822.

14 Spry ChJF. The pathogenesis of endomyocardial fibrosis: the role of the eosinophil. Springer Semin Immunopatho 1989;11:471-7.

15 Venge P. The human eosinophil in inflammation. Agents and Actions 1990;1/2:123-6.

16 Löffler W. Endocarditis parietalis fibroplastica mit Bluteosinophilie. Schw Med Wochenschr 1936;66:817-20.

17 Thüring Ch, Hess OM, Murakami T, et al. Normalwerte der linksventrikulären Funktion. Biplane Angiokardiogrpahie, unter Berücksichtigung geschlechtsspezifischer Unterschiede. Fortschr Röntgenstr 1989;150:562-8.

18 Roelandt JRTC, Sutherland GP, Iliceto S, et al. Cardiac ultrasound. Edinburgh: Churchill Livingstone, 1993.

19 Braunwald E. Heart disease Philadelphia: WB Saunders, 1997.

20 Liesveld JL, Abboud CN. State of the art: the hypereosinophilic syndromes. Blood Reviews 1991;5:29-37.

21 Jameson MD, Segraves StD. Idiopathic hypereosinophilic syndrome. Postgrad Med 1988;84:93-7.

22 Hess OM, Turina M, Egloff L, et al.Velauf der Endomyokardfibrose nach chirurgischer Endokarddekortikation. Schw Med Wochenschr 1984;114:1595-8.

23 Weller PF, Glenn BJ. The idiopathic hypereosinophilic syndrome. Blood 1994;10:2759-71. 